\title{
核酸一蛋白质互作的生物化学研究方法
}

张金璧，潘增祥，林飞，马雪山，刘红林

南京农业大学动物科技学院, 南京 210095

摘要: 研究核酸 - 蛋白质的互作是揭示生命活动机理的基础, 文章简要综述了用于研究核酸-蛋白质互作的 各种生物化学方法。从体内、体外两个研究角度, 针对核酸、蛋白以及复合物 3 个研究水平, 概述了硝化纤维 膜过滤实验、足迹法、EMSA、Southwestern 杂交等经典分析方法的原理、发展和运用。还着重介绍了最近在 表观遗传学领域中广泛运用的 nChIP、xChIP 等基本染色质免疫沉淀(ChIP)技术及其衍生出的 ChIP-on-chip 等 方法。

关键词：核酸; 蛋白质; 互作; 足迹; 染色质免疫沉淀

\section{Biochemical methods for the analysis of DNA-protein interactions}

\author{
ZHANG Jin-Bi, PAN Zeng-Xiang, LIN Fei, MA Xue-Shan, LIU Hong-Lin \\ College of animal science and technology, Nanjing Agricultural University, Nanjing 210095, China
}

\begin{abstract}
Investigation of DNA-protein interactions is fundamental to understand the mechanism underlying a variety of life processes. In this article, various types of biochemical methods in DNA-protein interaction study in vivo and in vitro at the level of DNA, protein, and the complex, respectively were briefly reviewed. Traditional assays including Nitrocellulose filter-binding assay, Footprinting, EMSA, and Southwestern blotting were summarized. In addition, chromatin immunoprecipitation techniques including nChIP, xChIP, and ChIP-on-chip, which were widely used in epigenetics, were particularly introduced.
\end{abstract}

Keywords: nucleic acid; protein; interaction; footprinting; chromatin immunoprecipitation (ChIP)

核酸 - 蛋白的互作在生命活动中发挥着广泛而 重要的作用，二者的协作是各种生命现象的基础。 将细胞或细胞器中的蛋白质、核酸等生物大分子的 相互作用联系起来, 是综合研究一个完整的生物学 途径的核心内容 ${ }^{[1]}$ 。近半个世纪以来, 研究者们在核 酸 - 蛋白质复合物的构成和分解过程中进行了大量 探索, 发展了一系列研究其互作关系的方法技术, 其中, 生物化学相关方法一直是重点与主流。
生物化学法主要利用酶或其他化学制剂, 通过 切割、修饰等作用来分析或分辨存在相互作用的核 酸和蛋白复合物, 研究其间潜在或实际的结合能力 和结合方式。其中 DNase I、Exo III 足迹法以及更加 精练的足迹技术，如羟基自由基足迹分析、保护/干 扰实验等，在鉴别潜在的 DNA 靶点的基础上可进一 步用于研究 DNA-蛋白复合物中的 DNA 构成; 消化 纤维膜过滤法、凝胶阻滞分析以及 Southwestern 印

收稿日期: 2008-07-18; 修回日期: 2008-12-08

基金项目: 国家重大科学研究计划项目(编号: 20007CB947403)和南京农业大学青年基金项目(编号: KJ07011)资助

作者简介: 张金璧(1985-), 女, 硕士研究生, 研究方向：动物遗传与发育生物学(表观遗传学方向)。Tel: 025-4395278; E-mail: 2007105010@njau.edu.cn

通讯作者: 刘红林(1966-), 教授, 博士生导师, 研究方向：胚胎发育与分子遗传学。Tel: 025-4395278; Fax: 025-4395314; E-mail: liuhonglin@njau.edu.cn 
迹等能用作结合位点确定后相关蛋白的分离分析; 随着表观遗传学的兴起, 建立在 DNA-蛋白交联基 础上的染色质免疫沉淀(Chromatin immunoprecipitation, ChIP)技术迅速发展起来，成为探索复杂的 DNA-蛋白复合物结合情况的重要手段, 被广泛运 用于转录复合体和组蛋白修饰的研究。本文就相关 方法的原理、应用及发展情况, 从体内、体外两个 方面进行综述。

\section{1 体外的生化研究方法}

在对于蛋白质 - 核酸互作的多数研究, 尤其是 早期研究中, 目的蛋白和目的核酸被分别提取出来, 在人工条件下进行孵育结合, 进行相关研究。虽然 实际上在生物体中, 二者有可能因为空间、电荷等 各种关系无法接近而产生作用, 但体外方法有效地 体现了核酸和蛋白质能够发生互作的潜力, 为核酸 蛋白质研究所广泛运用。

\section{1 硝化纤维膜过滤实验}

膜过滤方法在核酸 - 蛋白质复合物研究中渊源 已久, 最初用于RNA - 蛋白质的互作研究 ${ }^{[2]}$, 后由 Jones和Berg ${ }^{[3]}$ 于 1966 年首次将其用于DNA - 蛋白 质的研究中。其原理是蛋白与硝化纤维膜 (Nitrocellulose filter, NCF)结合, 但DNA不与其结 合。将标记过的DNA与蛋白质共同孵育, 用NCF过 滤混合物, 则DNA能够通过 NCF, 而DNA- protein 复合物留在NCF上。随后干燥 NCF, 通过标记物定量 分析留在滤膜上的复合物, 即可判断二者的结合程 度。

NC膜过滤的方法虽然在当前的研究中逐渐减少, 但其操作快速、简单, 能够定量地研究蛋白质与DNA 相互作用, 仍在分析较多个核酸 - 蛋白质的互作关系 中有一定用途。Tran等 ${ }^{[4]}$ 在蛋白互作研究中, 用膜过滤 法确定促旋酶的DNA结合特性, Haque等 ${ }^{[5]}$ 在研究线粒 体翻译起始因子与核糖体的互作关系时, 用此方法分 析起始复合物中RNA与核糖体蛋白的结合量变化情况; Posner等 ${ }^{[6]}$ 结合芯片作用原理, 用滤膜板一次性检验 384 个 $G$ 蛋白联合受体的可能结合部位。

\section{2 足迹法}

足迹法最大的特点在于能确定蛋白结合DNA的
片段长度, 其基本原理与DNA化学测序法相似, 首 先将待测双链DNA片段进行标记, 然后加入适当浓 度的探针对DNA进行消化剪切, 由于剪切具有随机 性，在反应完全时可将DNA切成单核苷酸。若控制 探针浓度, 将DNA部分消化, 就可以形成一个单核 甘酸、二核苷酸、三核苷酸……n核苷酸的混合物 ${ }^{[7]}$ 。 经变性后电泳分离, 放射自显影, 即可形成以相差 一个核苷酸为梯度的DNA条带。但当结合上蛋白时, 相应的DNA序列不会受探针的攻击, 因而在放射自 显影图谱上DNA梯度条带在相应DNA结合蛋白的 结合区域中断, 形成一空白区域, 恰似蛋白质在 DNA上留下的足迹, 因而被形象地称作足迹法。具 体原理见图 1 。

随着可选用的探针逐渐丰富, 足迹法的具体研 究方法和功能也丰富起来, 针对不同用途选取不同 的探针, 在解决多种实际问题中起到了很大作用。 用于互作分析的探针主要有两种: 生物酶探针和化 学探针, 以下分别进行介绍。

\subsection{1 酶足迹法}

生物酶探针特异性好, 一般不会与要研究的结合 蛋白作用, 不会扰乱DNA与蛋白质的互作。因此在脆 弱的DNA - 蛋白质复合体中, 酶探针更受欢迎 ${ }^{[8]}$ 。 DNase I 和exonuclease III 是两种最主要的生物酶探针, 在定位与蛋白质结合的DNA序列上有较大优势。

\subsubsection{DNaseI 足迹}

DNase I 是直径约 $40 \AA$ 的蛋白, 结合在DNA小 沟, 独立地切割两条链的磷酸骨架 ${ }^{[9]}$ 。由于其体积较 大, 切割作用更容易受空间位阻作用的影响, 不能 切割到有蛋白覆盖的区域及周边的DNA, 因此是足 迹法中确定DNA - 蛋白结合与否的理想方法之一, 可以确定结合在蛋白上的DNA的片段长度, 但不能 给出具体核酸序列。

DNase I 足迹法由Galas和Schmitz ${ }^{[7]}$ 于 1978 年 首次运用于DNA序列特异性结合蛋白的研究中。通 常是将DNA单链末端标记, 然后与结合蛋白反应, 复合物用DNase I 部分消化。结合蛋白的区域受到蛋 白保护, 免受DNase I 攻击, 而产物由于分子量的 差异, 经电泳和放射自显影即可得到一系列条带, 与对照消化产物的连续条带相比, 其中空缺部分即 为蛋白的结合区域。 

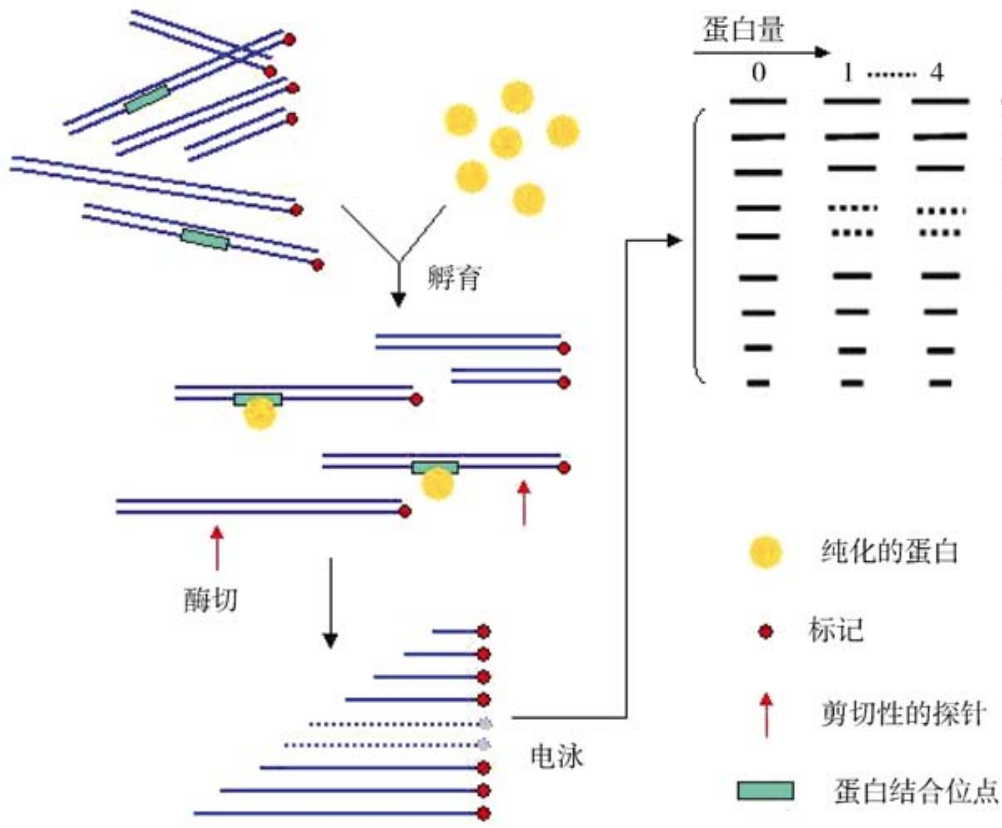

图 1 足迹法原理

通过同一DNA片段与多个蛋白的足迹分析，可 推断这些蛋白的结合域是否有交叠或相互分开，从 而推测这些蛋白之间的协作对基因的调节，如 Makarewicz等 ${ }^{[10]}$ 用单体PhoP进行DNase I 足纹法分 析得出, PhoP_P二聚体在phyC启动子上有两个结合 区域, 在启动子-35 位的结合促进启动子活性, 而在 -10 的结合抑制其活性; Matta等 ${ }^{[11]}$ 在拮抗酶AtoC和 细菌调控元件的互作研究中, 用DNase I 足迹法检 测AtoC的结合位点, 得出其在两个 $20 \mathrm{bp}$ 的位置结合, 序列分析得出这两个位置正好构成与转录起始位点 相关的回文序列。DNase I 足迹法在确定单一转录因 子结合区的研究中应用也很广泛。近期应用此法的 一个典型例子是 Connaghan-Jones 等 ${ }^{[12]}$ 在研究孕酮 受体与小鼠乳腺肿瘤病毒启动子的互作时, 采用 DNase I 足迹法检测出 $P R$ - $a$ 在启动子上的 5 个特异 结合区域。

当然, DNase I 法亦有其缺点。由于一些结合蛋 白也会与其发生作用, 导致一些 DNase I 超敏感位 点的存在。另外 DNase I 对 DNA 的切割有位点偏好 性, 因此得出的电泳梯度条带是不均匀的, 而且实 验中底物需要量大, 不能自动区别开 DNA - 蛋白质 复合体上的多个组分。这些不足使 DNase｜技术具 有一定局限性。

\subsubsection{2 外切酶 III 足迹法}

外切酶 III (Exonuclease III，Exo III)加工过程独特, 使其在研究序列特异性结合蛋白的工作中成为一种 常用的探针，适用于蛋白结合位置 DNA 序列的 分析。

Exo III 是单体酶, 分子量小 (28 $000 \mathrm{kDa})$, 具有 $3^{\prime} \rightarrow 5$ '外切酶活性、RNaseH活性、3'磷酸酶活性和 AP核酸内切酶活性 ${ }^{[13]}$ 。Exo III 足迹法运用了其 $3^{\prime} \rightarrow 5^{\prime}$ 外切酶活性 ${ }^{[14]}$, 当Exo III 切割双链DNA时, 有蛋白 结合的位置被保留下来, 产生的两条单链DNA片段 经过变性凝胶电泳分析, 放射自显影检测即可得到 结合片段大小。一般来说, ExollI 足迹的切割片段要 比DNase I 略小一些, 所有的未被结合的DNA都被 完全消化，这是Exo III 的优势所在，不存在自由 DNA产生的背景问题。不过使用ExolII探针的先决条 件是, 蛋白和DNA互作的半衰期不能少于Exo III作 用所需要的时间 ${ }^{[8]}$ 。

Exo III 足迹法最早由Shalloway等 ${ }^{[14]}$ 采用, 通过 此方法，他们用SV40 T抗体找出了SV40 DNA复制 起始位点结合区域。在新近的研究中仍受欢迎, 如 Wu等 ${ }^{[15]}$ 在对铂类抗癌药物的研究中用Exo III 足迹法 验证了铂类药物与核苷酸序列的结合情况，在药物 交联位置酶切明显受阻, 由此证实了铂类抗癌药在 
治疗中的可行性。Chen等 ${ }^{[16]}$ 将苂光染色技术和Exo III 足迹结合, 由于酶切DNA时激发了结合在探针上 的SYBR Green I的释放, 苂光强度在酶切和非酶切 位点显示出强弱差别并能被方便地检测出来, 这种 改进使得 Exo III 足迹法更快速敏感而且经济。Yang 等 ${ }^{[17]}$ 发现Exo III 并非只切割双链核甘酸, 将其与 a-thiotriphosphate以及聚合酶结合使用，可以分析生 物合成过程中多个大分子的协作行为, 对Exo III 的应 用进行了新的探索和拓展。

\subsection{2 化学足迹法}

理论上说, 能切断DNA骨架的化学试剂同样能 作为探针, 与酶探针相似, 当DNA与蛋白质结合时, 探针无法到达结合了蛋白的位点, 因此, 在化学探 针作用后再进行电泳, 这些没有被切断的位置将形 成空白条带。羟自由基足纹法 (Hydroxyl radical footprinting)即是其中应用较广泛的代表 ${ }^{[18]}$ 。

羟游离基足纹法是高分辨率的描绘DNA - 蛋白 质互作关系的方法。它利用芬顿反应产生的自由羟 基来攻击核糖骨架, 打开DNA主链, 从而获得足纹, 由于羟基作用点在沿着DNA分子表面排列的脱氧核 糖上，因此完全不受碱基的序列及种类的影响 ${ }^{[19]}$ ，当 DNA主链的脱氧核糖被蛋白质覆盖后，自由羟基无 法接近, 于是其产物在测序凝胶中出现若干区带强 度减弱的区域, 此即DNA主链与蛋白质的作用点。

与DNase I 和Exo III 足迹法不同, 羟游离基足纹法形 成的足迹更小，羟自由基只有水分子大小，不受空 间位阻约束, 而且切割均匀, 分辨率高, DNA分子主 链的全部位置可从足纹图一览无遗, 展现出更加详 细的信息。而且裂解效率高, 化学探针易于制备, 操 作简便。此法首先由Tullius等 ${ }^{[20]}$ 进行了总结, 在新 近研究中, Sanders等 ${ }^{[21]}$ 用Hydroxyl-radical足迹法成 功分析了 BPV initiator protein E1 在原核生物体中对 DNA的结合情况。

羟游离基足纹法的主要的局限在于自由羟基也 攻击蛋白，反应时间较长容易导致一些敏感蛋白的 降解, 扰乱原有的互作状态。对此, Shcherbakova等 ${ }^{[22]}$ 发明了一种“快速芬顿(Fast fenten)”的方法, 在毫 秒级的分辨率下研究大分子折叠, 互作和配合, 在 DNA、RNA和蛋白的瞬时作用上都可运用, 为解决 这一问题提供了新思路。

\subsection{3 保护实验/千扰实验}

此类方法先用化学试剂修饰DNA，再结合足迹 法, 可以得到碱基特异性结合位点相关的详细信 息。最常用的试剂有硫酸二甲酯(Dimethylsulphate, DMS，与嘌呤尤其是 $\mathrm{G}$ 作用) 和乙基亚硝基腿 (Ethylnitrosourea，与磷酸二酯键骨架作用)。由试剂 对DNA修饰和蛋白结合的时间不同分为两种，一般 来说先结合蛋白再修饰, 称为保护实验; 先修饰再 与蛋白结合, 称为干扰实验 ${ }^{[23]}$, 也有名称混用的 情况。

\subsubsection{1 甲基化(DMS)保护/千扰实验}

甲基化保护实验是研究蛋白与DNA双螺旋大沟 中的鸟嘌呤残基互作中最常用的方法, 也用于研究 与小沟中腺嘌呤的互作。DMS能与嘌呤快速反应, 生成N7-甲基鸟嘌呤或N3-甲基腺嘌呤。鸟嘌呤的N7 位于双螺旋的大沟中, 因此保护实验一般可以揭示 蛋白质侧链和特异碱基在大沟里的结合, 而腺嘌呤 的N3 位于双螺旋小沟内, 所以也可以指示出通过小 沟与DNA结合的蛋白 ${ }^{[24]}$ 。

本方法的基本步骤与DNase I 足迹法相似，结 果也类似, 但主要显示出了作用在 $\mathrm{G}$ 残基上的蛋白。 在保护实验中, 蛋白质先与标记过的DNA片段结合, 然后用DMS处理，DMS会使大沟中所有没有蛋白保 护的鸟嘌呤(以及小沟中没有蛋白保护的腺嘌呤)甲 基化, 随后在六氢吡啶(Piperidine)作用下被剪切。最 后将所得DNA片段用电泳分离, 放射自显影, 与先 前没有与蛋白孵育的DNA剪切条带进行对比, 即可 显示出空白的蛋白结合区域。此法在特异性结合的 蛋白研究中较常用, 如Flanigan等 ${ }^{[15]}$ 采用DMS保护 实验分析偏好在嘌呤碱基上结合的Gifsy-1 Xis蛋白 和Gifsy-1 attP序列的互作。

虽然保护技术在许多蛋白上运用顺利，但DMS 会直接作用于某些蛋白, 可能破坏其与DNA的结 合[26]。而且, 为了观察到足纹, 保护技术需要核酸与 蛋白质极紧密的结合 ${ }^{[27]}$, 这些局限性催生了第二种 技术一甲基化干扰实验 (Methylation interference assay)的出现。即先用DMS处理靶DNA, 使平均每条 链上只有一个 $\mathrm{G}$ 残基被甲基化。将局部甲基化的 DNA与含DNA结合蛋白的细胞提取物共同孵育, 结 合位点以及附近的DNA的修饰会阻止蛋白的结合。 
随后进行凝胶阻滞试验, 分离各种DNA条带, 并用 六氢吡啶切割甲基化的残基, 再与未结合蛋白的对 照一一对应进行电泳。如果因某个 $\mathrm{G}$ 残基的甲基化作 用而阻止了蛋白质与DNA的结合, 那么六氢吡啶对 这个甲基化G残基的切割作用就只能在没有蛋白质 结合的DNA中观察到。实验表明, 这是识别蛋白质 与特异碱基结合的分辨率较高的一种方 法 ${ }^{[28]}$ 。

\subsubsection{2 乙基化干涉实验}

原则上, 每一种化学探针试剂都可以用保护或 者干涉(预修饰)的方式进行实验, 但实际并非如此。 甲基化实验一般在DNA结合蛋白质后再进行甲基化 修饰，而乙基化实验则一般先用乙基亚硝基腿 (Ethylnitrosourea)修饰DNA, 再与蛋白结合, 修饰同 样会改变蛋白的结合程度。具体方法与甲基化干扰 实验相似, 但由于乙基化修饰作用于磷酸骨架, 相 对独立，不受碱基差异的影响，因此乙基化干扰实 验是少数几种不受碱基特异性干扰的研究方法之 一。另外, 乙基化干涉可以精确地鉴别DNA磷酸骨 架和蛋白接触的区域, Zhang等 ${ }^{229}$ 运用此方法精确定 位了Pura蛋白激活基因转录是通过和PDGF-A基因 启动子中富含 $\mathrm{G}$ 的单链区域结合来实现的。

化学足迹的方法也被统称为干扰技术或体内足 迹法, 其共同的优势在于可以研究蛋白质 - 核酸复 合体形成的分子细节, 而且在只有少量蛋白粗提物 的情况下也可以进行分析 ${ }^{[30]}$ 。所以, 其仍然是目前 较为广泛应用的技术方法。

\section{3 结合蛋白的定量分析法}

以上介绍的多种足迹方法都可以给出核酸 - 蛋 白复合体中核酸的长度或序列信息, 然而对于复合 物中的蛋白组分的分析还需要结合其他的分析方法, 才能对核酸和蛋白质综合分析, 描述其全面的互作情 况, 其中最为典型的方法是 EMSA 和 Southwestern 杂交。

\subsection{1电泳迁移率变动实验(Electrophoretic mobility shift assay, EMSA)}

电泳迁移率变动实验又称为凝胶阻滞实验 $(\mathrm{Gel}$ retardation assay)或凝胶移率变动实验 (Gel mobility shift), 是一种简单、快速和灵敏的用于体外检测蛋白 粗提取物中特异性 DNA 结合蛋白的技术。
EMSA的原理是DNA片段在聚丙烯酰胺凝胶电 泳中有一定迁移率，而蛋白质结合后，由于复合物 在体积, 形状, 电荷以及结合位置的不同, 迁移率 降低 [27]。传统方法用放射性标记的DNA探针(20 70 bp)与细胞提取物共孵育, 电泳后经放射自显影, 即 可把与蛋白质结合的探针和自由DNA探针从电泳胶 上分开。Fried等 ${ }^{[31]}$ 为我们提供了大量早期的EMSA 理论和实践的信息, Molloy等 ${ }^{[32]}$ 进一步阐述了较新 的具体方法和步骤。

EMSA操作过程简单、快速，灵敏度高 $\left(10^{-18} \mathrm{~mol}\right.$ $\left.\mathrm{DNA}^{[33]}\right)$, 可用于测定 DNA - 蛋白的结合参数, 从 而比较一个蛋白对几个相关DNA位点或者几个蛋白 对同一位点的亲和力 ${ }^{[34]}$ 。Zellner等 ${ }^{[35]}$ 用EMSA法检 测鼠科动物的pre-RC蛋白与DNA的结合情况, 比较 发现蛋白具有ATP依赖和位点特异性装配的特性。 Panno等 ${ }^{[36]}$ 将EMSA和ChIP平行使用, 从而确定了转 录因子 $S p 1$ 基因位点的 12 个序列参与了雌激素 $\mathrm{E} 2$ 诱 导的胰岛素受体底物IRS-1 表达上调。

随着标记技术的发展, 地高辛等非放射性标记 的采用, 避免了 ${ }^{32} \mathrm{P}$ 等标记探针有接触危险性且不容 易定量分析的缺点。而且在此基础上发展起来的毛 细管凝胶阻滞电泳技术, 结合了毛细管电泳的优势, 不需要使用放射性同位素, 样品用量少, 分辨率高, 解决了胚胎工程等研究中细胞数量限制的难题, 进 一步完善了凝胶阻滞电泳的方法 ${ }^{[37]}$ 。

\subsubsection{Southwestern 杂交}

DNA - 蛋白质印迹杂交, 即 Southwestern印迹 杂交(Southwestern Blot), 自 1980 年Bowen等 ${ }^{[38]}$ 创立 以来, 已广泛应用于分子遗传学、分子生物学的诸 多领域。将核蛋白粗提物进行SDS - PAGE电泳分析, 转膜后与同位素标记的特异DNA序列探针结合, 位 点特异性DNA结合蛋白借助于氢键、离子键和疏水 键结合DNA探针, 从而可通过放射自显影对DNA结 合蛋白进行定性、定量分析。最近, Harada1 等 ${ }^{[39]}$ 用 包含 CUX1 的双链核苷酸探针进行 SW 杂交, 分析 p110 CUX1 蛋白在DNA复制相关蛋白的编码基因中 的活化作用。

与前述方法不同的是, SW方法可以根据与标准 分子量大小的蛋白电泳谱相比较, 确定结合蛋白的 分子量。如Chen等 ${ }^{[40]}$ 为了描述JWA (-107/-28)启动 
子区的结合因子, 采用 SW杂交确定参与核酸 - 蛋 白质互作的分子, 将 anti-CBF/NF-Y、anti-NFI、 anti-CEBP, 以及 anti-GATA-1 抗体分别与同一张膜 孵育后, 发现只有anti-NFI显示有 $47 \mathrm{kDa}$ 的蛋白结 合作用。另外, 将SW和DNase I 或其他足迹法结合 使用可以达到同时检测结合蛋白大小和绘制其同源 结合域的双重功效 ${ }^{[41]}$ 。

SW 技术一定程度上要求经过 SDS - PAGE 电泳 后的蛋白复性, 不同蛋白的复性难易程度存在差别, 那些较难复性的蛋白附着在膜上后将丧失结合 DNA 的能力。而且, 那些需要几个亚基或辅因子共 同作用才有结合能力的蛋白也难以通过这种方法检 测到。

\section{2 体内研究方法}

染色质免疫沉淀 (Chromatin immunoprecipitation, ChIP) 是体内研究蛋白质 - DNA 互作强有力的 方法, 所谓体内 (in vivo)方法, 是直接将完整的细胞 进行交联处理，保持其内核酸和蛋白质的结合情况， 之后再对核酸和结合蛋白做进一步分析。此方法不 需要分别提取核酸或蛋白, 在天然的染色质环境下 进行，基本保持了二者在细胞内的真实结合状态。

ChIP方法最早广泛运用于酵母基因组的研究 中 ${ }^{[42]}$ 。在随后的十几年里, ChIP方法经过了研究者们 不断的摸索和改进, 迅速发展。随着表观遗传学的 兴起和发展, ChIP在染色体重塑的研究中崭露头角, 被运用于染色质相关的蛋白, 如组蛋白及其变异体, 特定DNA结构所结合的转录因子等研究中。ChIP的 基本操作由对染色质的处理方法不同分为非变性和 变性两种, 另外还根据不同实验需要产生了多种各 具特色的方法, 如Re-ChIP、ChIP Chop等。一般进行 完ChIP后直接用 qPCR 对DNA进行分析, 而近年来 ChIP与芯片技术结合, 展现出更广泛的 用途。

\section{1 非变性染色质免疫沉淀(nChIP)}

顾名思义, nChIP采用未变性的染色质进行实验, 用微球菌核酸酶(Micrococcal nuclease)来消化细胞 核, 使染色质消化成碎片, 随后用对应的抗体将结 合有蛋白质的染色质片段选择性地免疫沉淀下来, 再运用杂交或PCR方法进行DNA序列信息的分析。 这个方法来源于Dorbic和Wittig ${ }^{[43,44]}$ 的技术, O’Neill
等 ${ }^{[45]}$ 在实验室工作经验的基础上对此方法进行了总 结和步骤的规范化。

nChIP一般用于具有DNA高亲和力的组蛋白及 其变异体的研究中 $[46,47]$, 对于核心组蛋白修饰, 如 甲基化、乙酰化的分析最为合适。Bruce等 ${ }^{[48]}$ 采用 $n C h I P$ 检测了超乙酰化组蛋白变异体H2A.Z与看家 基因和组织特异基因在基因转录活化和非活化时的 结合情况。Huang等 ${ }^{[49]}$ 在白血病病理研究中用nChIP 检测了 GABAergic 基因启动子上的组蛋白甲基化情 况。在一些实验中也有用超声波破碎仪代替微球菌 核酸酶来破碎染色体，如Roca等 ${ }^{[50]}$ 对造骨细胞转录 因子及其结合位点的研究中的做法, 他们同时也提 出了nChIP的方法同样适用于Dlx蛋白和 Sin $3 \mathrm{~A}$ 等与 DNA高亲和力结合的非组蛋白。考虑到一些转录因 子与DNA的结合造成空间位阻，会导致nChIP得到 的组蛋白结合量偏少, Brand等在染色质破碎后用羟 基磷灰石(Hydroxyapatite, HAP)纯化核小体，先去除 其他蛋白再进行免疫沉淀, 提高了nChIP的精确性 [51]。为了突破底物量的限制(一般需要 $10^{6} \sim 10^{7}$ 以上 数量级的细胞), O'Neill等 ${ }^{[52]}$ 还在对小鼠胚胎的研究 中采用了果蝇细胞作为载体, “稀释” 小鼠细胞, 仅 用 50 100 个细胞成功进行了 nChIP, 并命名为 “carrier” ChIP(CChIP), 使ChIP技术的发展迈进了 一大步。2007 和 2008 年, Dahl等 ${ }^{[53,54]}$ 先后提出了针 对少量细胞操作的 $\mathrm{Q}^{2} \mathrm{ChIP}$ 和进一步改进后的 $\mu$ ChIP 的实验方法。

nChIP 在分析核酸 - 蛋白质互作中具有明显的 优势, 首先, 微球菌核酸酶消化后的染色质约为 $1 \sim 5$ 个核小体大小, 可以通过蔗糖梯度离心来分离出单 个核小体核小体，选用单核小体作为实验用的染色 质, 可以实现特定位点 DNA 或者特定为点上的蛋白 质在核小体水平上的定位, 比变性 ChIP 更为精确。 同时，没有交联剂的处理，降低了抗原被削弱和修 饰的可能。但 nChIP 方法难以检测到与核酸结合较 弱的蛋白, 如转录因子等。

\section{2 变性(交联)染色质免疫沉淀(xChIP)}

由于可以在大量与DNA联系不太紧密的染色质 结合因子的研究中使用, xChIP比nChIP的应用更加 广泛。其方法是先将染色质进行变性处理, 一般采 用向细胞中加入甲醛 $[55,56]$, 或者将细胞暴露在紫外 
线下的方法。使DNA和蛋白质发生交联, 接着用超 声波将这些染色质切割成小片段, 用特异性蛋白质 抗体来选择性地免疫沉淀蛋白质 - DNA复合物。最 后逆转交联, 进一步分析沉淀下来的DNA。具体流 理见图 2。

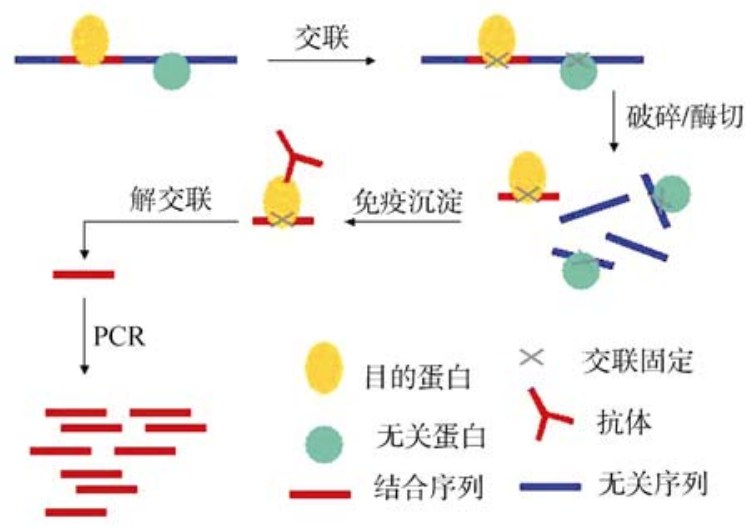

图 2 变性染色质免疫沉淀流理

xChIP适用于分析与DNA结合能力较弱的蛋白 (连接组蛋白和大多数非组蛋白), 一个典型的例子 是 Alcorlo 等 ${ }^{[57]}$ 用 xChIP 检测抗菌素GA-1 蛋白与 DNA的结合情况。由于xChIP与nChIP分别在非组蛋 白和组蛋白的研究方面具有优势, 形成互补, 可根 据实际实验的需要灵活选择。Han等 ${ }^{[58]}$ 在对CTCF的 研究中分别采用N-ChIP和 X-ChIP分析组蛋白共价 修饰情况并检测macroH2A1 组蛋白变异体; Mann等 [59]在其研究中也同时采用两种ChIP方法, 其目的是 验证和确定抑制蛋白 $\mathrm{I} \chi \mathrm{B} \alpha$ 和氧化物酶体增生活化受 体 $\gamma(\operatorname{PPAR} \gamma)$ 启动子上蛋白的结合。

甲醛是 XChIP中最为常用的交联剂, 在其作用 下，DNA碱基上的氨基或亚氨基和蛋白质上的a氨基 及赖氨酸、精氨酸、组氨酸、色氨酸的侧链氨基会 与其他DNA或蛋白质上的氨基或亚氨基交联在一起, 在几分钟内形成生物复合体。其优势在于, 甲醛并 不作用于游离的双链DNA, 从而避免了DNA损伤, 另外, 醛交联反应是完全可逆的, 便于在后续步骤 中分别对DNA和蛋白质进行分析。值得注意的是, 交联过程中，一些抗原决定基可能发生改变或闭塞， 某些抗原本身难以用xChIP方法进行共沉淀。另外, 由于不能预测有多少抗原以及多少域点能真正与 DNA结合，也存在某一位点之间本身存在的结合难
易程度不同, 因此X-ChIP无法对蛋白定 量 ${ }^{[60]}$ 。 nChIP 和 xChIP 的方法各有其优势和局限, 总 的来说可概括如表 1 。

表 1 nChIP 和 XChIP 的差别

\begin{tabular}{lll}
\hline & \multicolumn{1}{c}{ nChIP } & \multicolumn{1}{c}{ xChIP } \\
\hline 交联处理 & $\begin{array}{l}\text { 不用交联剂或短时 } \\
\text { 间交联 }\end{array}$ & $\begin{array}{l}\text { 需要甲醛或紫外线等处 } \\
\text { 理 } 10 \mathrm{~min} \text { 以上 }\end{array}$ \\
染色质破碎 & 一般采用微球菌酶 & 一般采用超声波破碎仪 \\
研究对象 & 高亲和力蛋白(组蛋白 & 低亲和力蛋白(连接组蛋 \\
& 及其变异体等) & 白、转录因子等) \\
DNA 序列分析 & 易(酶切位点固定) & 难(破碎的片段长度不一) \\
蛋白定量 & 易 & 难 \\
精确度 & 单核小体水平 & 染色质水平 \\
沉淀效率 & 较高 & 较低(受抗原决定基影响) \\
in vivo 程度 & 较低(过程中核小体 & 较高(核小体重序的可能 \\
& 可能出现重序) & 性最低) \\
\hline
\end{tabular}

\subsection{Re-ChIP}

Re-ChIP 法对同一 DNA 底物, 运用两种不同的 抗体, 相继进行了两次免疫共沉淀, 可以确定两种 抗原，即两种结合蛋白在同一染色质片段上的结 合。这种方法为 DNA 结合的蛋白复合体的研究提供 了新的思路, 让我们能更清晰地描绘蛋白的结合次 序和相互协作关系。

Re-ChIP法在DNA结合的多蛋白复合物, 尤其 是转录复合体的分析研究中得到了充分运用, 一般 将ChIP和Re-ChIP连续使用, 分别用两种抗体与相 同DNA底物进行沉淀。在启动子结合因子的研究中, Gaughan等 ${ }^{[61]}$ 运用Re-ChIP方法, 确定PSA基因启动 子上结合的蛋白多聚体中包含 AR 和 HDAC1。 Wissmann等 ${ }^{[62]}$ 先后用雄激素受体抗体和JMJD2C抗 体进行ChIP, 证明了雄激素受体和JMJD2C都在PSA 启动子上结合形成蛋白复合体。Sayeed等 ${ }^{[63]}$ 分别用 $\mathrm{ERa}$ 和p53 的抗体进行免疫共沉淀, 发现两者都在 Survivin和 MDR1 的启动子上p53 结合区出现, 而 $\mathrm{ERa}$ 的这种共结合对p53 的功能有抑制作用。非启动 子序列的研究中, Re-ChIP也同样适用, Schnekenburger等 ${ }^{[64]}$ 采用Re-ChIP方法揭示了Deacetylase 1 和 DNA Methyltransferase 1 复合体与染色体的结合是 转录活性改变的重要标志。Wilkinson等 ${ }^{[65]}$ 对p53 和 Smad蛋白在SBE/p53RE位置上的结合进行研究，揭 
示转录生长因子介导的转录抑制作用。

要注意的是, 非同一复合体上的两个蛋白也可 能都与底物片段结合, 因此 Re-ChIP 方法的结果也 不能作为确定蛋白复合体提供绝对的证据。

\subsection{ChIP Chop}

ChIP Chop是一另项建立在ChIP基础上的方法, 可以检测蛋白结合的DNA位置的甲基化情况。在实 际的染色质免疫沉淀实验中, $5 \mathrm{meC}$ 的抗体无法共沉 淀甲基化DNA，常导致甲基化DNA的共沉淀无法顺 利进行。为了解决这个问题, Lawrence等 ${ }^{[66]}$ 工妙运用 了核苷三磷酸盐依赖的限制性内切酶McrBC, 即等 在进行过ChIP后, 用McrBC酶水解DNA, 随后再用 跨越目的序列的引物进行PCR扩增。McrBC酶的特 点是特异性剪切含有两个以上甲基化 $\mathrm{C}$ 二核甘酸的 DNA ${ }^{[67]}$, 如果DNA模版超甲基化, 则被McrBC酶切 断, 因此无法扩增出目的区段。相反，低甲基化的模 版对M crBC酶具有抵抗力, 则能扩增出目的片段。 通过这种方法便可以确定出DNA结合抗原的位置是 否发生了严重的甲基化。

此方法自提出后得到了许多研究者的亲睐, Preuss 等 ${ }^{[68]}$ 在 rRNA 基因沉默的研究中运用 ChIP Chop, 评估结合了蛋白的DNA序列上胞嘧啶的甲基 化密度。Earley等 ${ }^{[69]}$ 采用此方法检测组蛋白结合的 rRNA基因启动子上胞嘧啶去甲基化变化。

2.5 ChIP-on-chip (Chromatin immuno-precipitation based on microarray)

ChIP-on-chip 是一种全基因组范围内的定位分 析技术，建立于染色质免疫沉淀(ChIP)和芯片技术 (Chip)的联合运用之上。用于分析细胞中 DNA 结合 蛋白对特异结合位点, 包括启动子、增强子、抑制 子、沉默子、绝缘子、边界元件, 以及 DNA 复制的 调控序列的鉴定, 整体研究生物体发育和病变过程 中的复杂信息网络, 是一个绘制基因组功能元件作 用网络的技术平台。

对此技术的成功运用最早可追溯到 2000 2001 年的 3 篇文章中 ${ }^{[70 ~ 72]}$, 随后, ChIP-on-chip技术迅速 发展并得以广泛应用 2002 年, Richard Young的团 队 [73]用c-Myc tagging system确定了酵母 106 个转录 因子的全基因组的结合位点, 同年, Weinmann等 ${ }^{[74]}$ 用其篮选转录因子的未知靶序列。自此,
ChIP-on-chip开始成为全基因组水平描述组蛋白和 许多调控因子作用情况的强大工具。通过对 ChIP-on-chip方法的运用, Testa等 ${ }^{[75]}$ 揭示了 NF-Y蛋 白在核心启动子之外的多个的CCAAT结合位点, Liu 等 ${ }^{[76]}$ 在染色体组水平上全面检测了 RE-a和ER-b的 结合位点。Wendt等 ${ }^{[77]}$ 在最近发表的文章中全面运用 了ChIP-on-chip技术，在全基因组水平上检测了不 同细胞时期的黏着素结合位点, 还对CTCF sites位 点在多个细胞系的分布做了描述, 其中运用了 Affymetrix arrays, ENCODE arrays 等不同的 ChIP-on-chip类型，芯片装载的DNA片段长度也各 不相同, 可谓将ChIP-on-chip运用到了极致。

由于哺乳动物基因组的庞大和高重复性, 过去 的研究一般都集中于对候选因子功能的确定而非全 基因组的分析，然而随着近年来 Nimblegen 等公司 的哺乳动物基因组芯片的问世, 研究者不需要预先 考虑蛋白质的可能结合位点, 对哺乳动物在全基因 组水平上进行高分辨率的 DNA 结合蛋白, 染色质构 成等研究也就成为可能。

ChIP-on-chip 作为一种新技术, 也有一些缺陷 尚待改善。首先是成本较高, 一般公开发表的运用 ChIP 方法的研究中都至少重复 3 次以保证其可靠性, 其高昂的成本成为一些实验室运用此方法的限制因 素; 其次, 对芯片实验得到的大量数据进行统计和 分析, 规范实验程序, 制定确切的有生物学意义的 实验标准等方面也尚待完善; 另一个限制因素是所 能得到的 DNA 片段的大小，一般的超声波裂解只能 得到约 $200 \mathrm{bp}$ 的片段。但为了得到高分辨率的结果, 需要得到更小的甚至单核苷酸的片段, 目前公司提 供的核苷酸序列从 70 bp (Microarrays, Inc.)到 25 bp(Affymetrix)不等。ChIP-on-chip 所需的抗体制备 也是一个障碍, ChIP-on-chip 要求特异性很高的抗体, 要在自由溶液和固定液中都能识别其抗原决定簇 (Epitope)。为了克服特异性的问题, 目的蛋白可与 FLAG 或 HA 等标签结合, 而 FLAG 或 HA 等能被抗 体所识别, 而且, 目前亦可以获得抗某一特异性组 蛋白修饰的抗体, 如 H3K4me3 等, 这些抗体和 ChIP-on-chip 的结合为组蛋白密码的破解和表观遗 传学的发展打开了一扇大门。

需要说明的是, 之所以称 ChIP 为体内的研究方 法, 主要是由于其相对体外方法而言, ChIP 方法没 
有分别提取出核酸或蛋白, 而是直接对完整的细胞 进行处理, 相对地保持了细胞内的核酸 - 蛋白质的 原始互作状态。但其结果也具有一系列不确定因素: 首先，生物体内的核酸，蛋白之间的互作具有时间、 空间的多样性, 瞬时的互作可能难以捕捉, 而偶然 的靠近也可能被固定下来; 第二, 在交联、酶切、超 声波破碎或其他处理过程中，核小体乃至染色质的 状态都可能受到化学和机械的影响而发生改变。

我们旨在对研究核酸 - 蛋白相互作用的相关技 术，尤其是新技术进行概括与综述，同时以最新的 研究例证作为代表进行介绍与讨论, 以期为读者进 行相关研究提供有益的思路或线索; 因为篇幅的限 制, 我们也不得不省略一些较传统的研究方法, 如 化学修饰与限制性蛋白水解、DNA 结合蛋白及其功 能域的过表达技术等。总之，随着生物化学方法的 进一步发展以及化学、物理学、计算机科学、信息 科学等学科的交叉协作, 核酸 - 蛋白质的研究方法 将不断丰富和完善。相信在科学工作者们的不解努 力下，对核酸 - 蛋白的互作机制的探究将更加深入, 对生命现象本质的理解将不断推进。

\section{参考文献(References):}

[1] Guille MJ, Kneale GG. Methods for the analysis of DNA-protein interactions. Mol Biotechnol, 1997, 8(1): 35-52.[DOI]

[2] Nirenberg M, Leder P. Rna Codewords and protein synthesis the effect of trinucleotides upon the binding of sRNA to ribosomes. Science, 1964, 145: 1399-1407.[DOI]

[3] Jones OW, Berg P. Studies on the binding of RNA polymerase to polynucleotides. J Mol Biol, 1966, 22(2): 199-209.[DOI]

[4] Tran JH, Jacoby GA, Hooper DC. Interaction of the plasmid-encoded quinolone resistance protein QnrA with Escherichia coli topoisomerase IV. Antimicrob Agents Chemother, 2005, 49(7): 3050-3052.[DOI]

[5] Haque ME, Grasso D, Spremulli LL. The interaction of mammalian mitochondrial translational initiation factor 3 with ribosomes: evolution of terminal extensions in IF3mt. Nucleic Acids Res, 2008, 36(2): 589-597.

[6] Posner B, Hong Y, Benvenuti E, Potchoiba M, Nettleton D, Lui L, Ferrie A, Lai F, Fang Y, Miret J, Wielis C, Webb B. Multiplexing $G$ protein-coupled receptors in microarrays: a radioligand-binding assay. Anal Biochem, 2007, 365(2): 266-273.[DOI]
[7] Galas DJ, Schmitz A. DNase footprinting: a simple method for the detection of protein-DNA binding specificity. Nucleic Acids Res, 1978, 5(9): 3157-3170.[DOI]

[8] Metzger W, Heumann H. Footprinting with Exonuclease III. DNA-Protein Interactions: Principles and Protocols, 1994, 30: 11-20.[DOI]

[9] Suck D, Lahm A, Oefner C. Structure refined to 2 Aa of a nicked DNA octanucleotide complex with DNase I. Nature, 1988, 332(6163): 464-468.[DOI]

[10] Makarewicz O, Dubrac S, Msadek T, Borriss R. Dual role of the PhoP approximately $\mathrm{P}$ response regulator: Bacillus amyloliquefaciens FZB45 phytase gene transcription is directed by positive and negative interactions with the phyC promoter. $J$ Bacteriol, 2006, 188(19): 6953-6965.[DOI]

[11] Matta MK, Lioliou EE, Panagiotidis CH, Kyriakidis DA, Panagiotidis CA. Interactions of the antizyme AtoC with regulatory elements of the Escherichia coli atoDAEB operon. J Bacteriol, 2007, 189(17): 6324-6332.[DOI]

[12] Connaghan-Jones KD, Heneghan AF, Miura MT, Bain DL. Thermodynamic dissection of progesterone receptor interactions at the mouse mammary tumor virus promoter: monomer binding and strong cooperativity dominate the assembly reaction. $J$ Mol Biol, 2008, 377(4): 1144-1160.[DOI]

[13] Rogers SG, Weiss B. Exonuclease III of Escherichia coli K-12, an AP endonuclease. Methods Enzymol, 1980, 65(1): 201-211.[DOI]

[14] Shalloway D, Kleinberger T, Livingston DM. Mapping of SV40 DNA replication origin region binding sites for the SV40 $\mathrm{T}$ antigen by protection against exonuclease III digestion. Cell, 1980, 20(2): 411-422.[DOI]

[15] Wu B, Droge P, Davey CA. Site selectivity of platinum anticancer therapeutics. Nat Chem Biol, 2008, 4(2): 110-112.[DOI]

[16] Chen Z, Ji M, Hou P, Lu Z. Exo-Dye-based assay for rapid, inexpensive, and sensitive detection of DNA-binding proteins. Biochem Biophys Res Commun, 2006, 345(3): 1254-1263.[DOI]

[17] Yang Z, Sismour AM, Benner SA. Nucleoside alpha-thiotriphosphates, polymerases and the exonuclease III analysis of oligonucleotides containing phosphorothioate linkages. Nucleic Acids Res, 2007, 35(9): 3118-3127.[DOI]

[18] Tullius TD. DNA footprinting with hydroxyl radical. Nature, 1988, 332(6165): 663-664.[DOI]

[19] Zaychikov E, Schickor P, Denissova L, Heumann H. Hydroxyl radical footprinting. Methods Mol Biol, 2001, 148: 
49-61.

[20] Tullius TD, Dombroski BA, Churchill ME, Kam L. Hydroxyl radical footprinting: a high-resolution method for mapping protein-DNA contacts. Methods Enzymol, 1987, 155: 537-558.[DOI]

[21] Sanders CM. A DNA-binding activity in BPV initiator protein E1 required for melting duplex ori DNA but not processive helicase activity initiated on partially single-stranded DNA. Nucleic Acids Res, 2008, 36(6): 1891-1899.[DOI]

[22] Shcherbakova I, Brenowitz M. Monitoring structural changes in nucleic acids with single residue spatial and millisecond time resolution by quantitative hydroxyl radical footprinting. Nat Protoc, 2008, 3(2): 288-302.[DOI]

[23] Vigneault F, Guerin SL. Regulation of gene expression: probing DNA-protein interactions in vivo and in vitro. Expert Rev Proteomics, 2005, 2(5): 705-718.[DOI]

[24] Baldwin AS. Methylation interference assay for analysis of DNA-protein interactions. Currt Protoc Mol Biol, 1988, 2: $1-12.13$

[25] Flanigan A, Gardner JF. Interaction of the Gifsy-1 Xis protein with the Gifsy-1 attP sequence. J Bacteriol, 2007, 189(17): 6303-6311.[DOI]

[26] Fairall L, Rhodes D, Klug A. Mapping of the sites of protection on a $5 \mathrm{~S}$ RNA gene by the Xenopus transcription factor IIIA. A model for the interaction. J Mol Biol, 1986, 192(3): 577-591.[DOI]

[27] Carey MF, Smale ST. Transcriptional Regulation in Eukaryotes: Concepts, Strategies, and Techniques. Cold Spring Harbor Laboratory Press, 2000.

[28] Shaw PE, Stewart AF. Identification of protein-DNA contacts with dimethyl sulfate. methylation protection and methylation interference. Methods Mol Biol, 2001, 148: 221-227.

[29] Zhang Q, Pedigo N, Shenoy S, Khalili K, Kaetzel DM. Puralpha activates PDGF-A gene transcription via interactions with a G-rich, single-stranded region of the promoter. Gene, 2005, 348: 25-32.[DOI]

[30] Damante G, Fabbro D, Pellizzari L, Civitareale D, Guazzi S, Polycarpou-Schwartz M, Cauci S, Quadrifoglio F, Formisano S, Di Lauro R. Sequence-specific DNA recognition by the thyroid transcription factor-1 homeodomain. $\mathrm{Nu}$ cleic Acids Res, 1994, 22(15): 3075-3083.[DOI]

[31] Fried MG, Daugherty MA. Electrophoretic analysis of multiple protein-DNA interactions. Electrophoresis, 1998, 19(8-9): 1247-1253.[DOI]

[32] Molloy PL. Electrophoretic mobility shift assays. Methods
Mol Biol, 2000, 130: 235-246.

[33] Rousseau S, Renaud J, Ruiz-Carrillo A. Basal expression of the histone $\mathrm{H} 5$ gene is controlled by positive and negative cis-acting sequences. Nucleic Acids Res, 1989, 17(18): 7495-7511.[DOI]

[34] Fried MG. Measurement of protein-DNA interaction parameters by electrophoresis mobility shift assay. Electrophoresis, 1989, 10(5-6): 366-376.[DOI]

[35] Zellner E, Herrmann T, Schulz C, Grummt F. Site-specific interaction of the murine pre-replicative complex with origin DNA: assembly and disassembly during cell cycle transit and differentiation. Nucleic Acids Res, 2007, 35(20): 6701-6713.[DOI]

[36] Panno ML, Marsico S, Bellizzi D, Rizza P, Morelli C, Salerno M, Giordano F, and Ando' S. Evidence that the mouse insulin receptor substrate-1 belongs to the gene family on which the promoter is activated by estrogen receptor through its interaction with Sp1. J Mol Endocrinol, 2006, 36: 91-105.[DOI]

[37] LeCaptain DJ, Michel MA, Orden AV. Characterization of DNA-protein complexes by capillary electrophoresissingle molecule fluorescence correlation spectroscopy. Analyst, 2001, 126(8): 1279-1284.[DOI]

[38] Bowen B, Steinberg J, Laemmli UK, Weintraub H. The detection of DNA-binding proteins by protein blotting. Nucleic Acids Res, 1980, 8(1): 1-20.[DOI]

[39] Harada R, Vadnais C, Sansregret L, Leduy L, Berube G, Robert F, Nepveu A. Genome-wide location analysis and expression studies reveal a role for p110 CUX1 in the activation of DNA replication genes. Nucleic Acids Res, 2008, 36(1): 189-202.

[40] Chen R, Qiu W, Liu Z, Cao X, Zhu T, Li A, Wei Q, Zhou J. Identification of JWA as a novel functional gene responsive to environmental oxidative stress induced by benzo[a]pyrene and hydrogen peroxide. Free Radic Biol Med, 2007, 42(11): 1704-1714.[DOI]

[41] Smith SE, Papavassiliou AG. A coupled Southwestern-DNase I footprinting assay. Nucleic Acids Res, 1992 20(19): 5239-5240.[DOI]

[42] Braunstein M, Rose AB, Holmes SG, Allis CD, Broach JR. Transcriptional silencing in yeast is associated with reduced nucleosome acetylation. Genes Dev, 1993, 7(4): 592-604.[DOI]

[43] Dorbic T, Wittig B. Isolation of oligonucleosomes from active chromatin using HMG17-specific monoclonal antibodies. Nucleic Acids Res, 1986, 14(8): 3363-3376.[DOI] 
[44] Dorbic T, Wittig B. Chromatin from transcribed genes contains HMG17 only downstream from the starting point of transcription. EMBO J, 1987, 6(8): 2393.

[45] O'Neill LP, Turner BM. Immunoprecipitation of native chromatin: NChIP. Methods, 2003, 31(1): 76-82.[DOI]

[46] Hebbes TR, Thorne AW, Crane-Robinson C. A direct link between core histone acetylation and transcriptionally active chromatin. EMBO J, 1988, 7(5): 1395-1402.

[47] O'Neill LP, Turner BM. Immunoprecipitation of native chromatin: NChIP. Methods, 2003, 31(1): 76-82.[DOI]

[48] Bruce K, Myers FA, Mantouvalou E, Lefevre P, Greaves I, Bonifer C, Tremethick DJ, Thorne AW, Crane-Robinson C, Journals O. The replacement histone H2A. Z in a hyperacetylated form is a feature of active genes in the chicken. Nucleic Acids Res, 2005, 33(17): 5633-5639.[DOI]

[49] Huang HS, Matevossian A, Whittle C, Kim SY, Schumacher A, Baker SP, Akbarian S. Prefrontal dysfunction in schizophrenia involves mixed-lineage leukemia 1-regulated histone methylation at GABAergic gene promoters. J Neurosci, 2007, 27(42): 11254.[DOI]

[50] Roca H, Franceschi RT. Analysis of transcription factor interactions in osteoblasts using competitive chromatin immunoprecipitation. Nucleic Acids Res, 2008,

[51] Brand M, Rampalli S, Chaturvedi CP, Dilworth FJ. Analysis of epigenetic modifications of chromatin at specific gene loci by native chromatin immunoprecipitation of nucleosomes isolated using hydroxyapatite chromatography. Nat Protoc, 2008, 3(3): 398-409. [DOI]

[52] O'Neill LP, VerMilyea MD, Turner BM. Epigenetic characterization of the early embryo with a chromatin immunoprecipitation protocol applicable to small cell populations. Nat Genet, 2006, 38(7): 835-841.[DOI]

[53] Dahl JA, Collas P. Q2ChIP, a quick and quantitative chromatin immunoprecipitation assay, unravels epigenetic dynamics of developmentally regulated genes in human carcinoma cells. Stem Cells, 2007, 25(4): 1037.[DOI]

[54] Dahl JA, Collas P. $\mu$ ChIP--a rapid micro chromatin immunoprecipitation assay for small cell samples and biopsies. Nucleic Acids Res, 2008, 36(3): e15.

[55] Solomon MJ, Varshavsky A. Formaldehyde-mediated DNAprotein crosslinking: a probe for in vivo chromatin structures. Proc Natl Acad Sci USA, 1985, 82(19): 6470-6474.[DOI]

[56] Solomon MJ, Larsen PL, Varshavsky A. Mapping protein-DNA interactions in vivo with formaldehyde: evidence that histone $\mathrm{H} 4$ is retained on a highly transcribed gene. Cell, 1988, 53(6): 937-947.[DOI]

[57] Alcorlo M, Salas M, Hermoso JM. In vivo DNA binding of bacteriophage GA-1 protein p6? J Bacteriol, 2007, 189(22): 8024-8033.[DOI]

[58] Han L, Lee DH, Szabó PE. CTCF is the master organizer of domain-wide allele-specific chromatin at the H19/Igf2 imprinted region? Mol Cell Bio, 2008, 28(3): 1124-1135.

[59] Mann J, Oakley F, Akiboye F, Elsharkawy A, Thorne AW, Mann DA. Regulation of myofibroblast transdifferentiation by DNA methylation and MeCP2: implications for wound healing and fibrogenesis. Cell Death Differ, 2007, 14: 275-285.[DOI]

[60] Orlando V. Mapping chromosomal proteins in vivo by formaldehyde-crosslinked-chromatin immunoprecipitation. Trends Biochem Sc, 2000, 25(3): 99-104.

[61] Gaughan L, Logan IR, Neal DE, Robson CN. Regulation of androgen receptor and histone deacetylase 1 by Mdm2-mediated ubiquitylation. Nucleic Acids Res, 2005, 33(1): 13-26.[DOI]

[62] Wissmann M, Yin N, Müller JM, Greschik H, Fodor BD, Jenuwein T, Vogler C, Schneider R, Günther T, Buettner R. Cooperative demethylation by JMJD2C and LSD1 promotes androgen receptor-dependent gene expression. Nat Cell Biol, 2007, 9: 347-353.[DOI]

[63] Sayeed A, Konduri SD, Liu W, Bansal S, Li F, Das GM. Estrogen receptor \{alpha\} inhibits p53-mediated transcriptional repression: implications for the regulation of apoptosis. Cancer Res, 2007, 67(16): 7746.[DOI]

[64] Schnekenburger M, Talaska G, Puga A. Chromium cross-links histone deacetylase 1-DNA methyltransferase 1 complexes to chromatin, inhibiting histone-remodeling marks critical for transcriptional activation? Mol Cell Biol, 2007, 27(20): 7089-7101.[DOI]

[65] Wilkinson DS, Tsai WW, Schumacher MA, Barton MC. Chromatin-bound p53 anchors activated Smads and the mSin3A corepressor to confer TGF \{beta\}-mediated transcription repression. Mol Cell Biol, 2008, 28(6): 1988-1998.[DOI]

[66] Lawrence RJ, Earley K, Pontes O, Silva M, Chen ZJ, Neves N, Viegas W, Pikaard CS. A concerted DNA methylation/histone methylation switch regulates rRNA gene dosage control and nucleolar dominance. Mol Cell, 2004, 13(4): 599-609.[DOI]

[67] Dryden DTF, Murray NE, Rao DN, Journals O. Nucleoside triphosphate-dependent restriction enzymes. Nucleic Acids Res, 2001, 29(18): 3728-3741.[DOI]

[68] Preuss S, Pikaard CS. rRNA gene silencing and nucleolar dominance: insights into a chromosome-scale epigenetic on/off switch. BBA-Gene Struct Expr, 2007, 1769(5-6): 
383-392.

[69] Earley K, Lawrence RJ, Pontes O, Reuther R, Enciso AJ, Silva M, Neves N, Gross M, Viegas W, Pikaard CS. Erasure of histone acetylation by Arabidopsis HDA6 mediates large-scale gene silencing in nucleolar dominance. Genes Dev, 2006, 20(10): 1283-1293.[DOI]

[70] Lieb JD, Liu X, Botstein D, Brown PO. Promoter-specific binding of Rap1 revealed by genome-wide maps of protein-DNA association. Nat Genet, 2001, 28(4): 327-334.[DOI]

[71] Ren B, Robert F, Wyrick JJ, Aparicio O, Jennings EG, Simon I, Zeitlinger J, Schreiber J, Hannett N, Kanin E, Volkert TL, Wilson CJ, Bell SP, Young RA. Genome-wide location and function of DNA binding proteins. Science, 2000, 290(5500): 2306-2309.[DOI]

[72] Iyer VR, Horak CE, Scafe CS, Botstein D, Snyder M, Brown PO. Genomic binding sites of the yeast cell-cycle transcription factors SBF and MBF. Nature, 2001, 409 (6819): 533-538.[DOI]

[73] Lee TI, Rinaldi NJ, Robert F, Odom DT, Bar-Joseph Z, Gerber GK, Hannett NM, Harbison CT, Thompson CM, Simon I, Zeitlinger J, Jennings EG, Murray HL, Gordon
DB, Ren B, Wyrick JJ, Tagne JB, Volkert TL, Fraenkel E, Gifford DK, Young RA. Transcriptional regulatory networks in Saccharomyces cerevisiae. Science, 2002, 298 (5594): 799-804.[DOI]

[74] Weinmann AS, Farnham PJ. Identification of unknown target genes of human transcription factors using chromatin immunoprecipitation. Methods, 2002, 26(1): 37-47.[DOI]

[75] Testa A, Donati G, Yan P, Romani F, Huang THM, Viganò MA, Mantovani R. Chromatin immunoprecipitation (ChIP) on chip experiments uncover a widespread distribution of NF-Y binding CCAAT sites outside of core promoters. $J$ Biol Chem, 2005, 280(14): 13606-13615.[DOI]

[76] Liu Y, Gao H, Marstrand TT, Strom A, Valen E, Sandelin A, Gustafsson JA, Dahlman-Wright K. The genome landscape of ER \{alpha\}-and ER \{beta\}-binding DNA regions. Proc Natl Acad Sci USA, 2008, 105(7): 2604.[DOI]

[77] Wendt KS, Yoshida K, Itoh T, Bando M, Koch B, Schirghuber E, Tsutsumi S, Nagae G, Ishihara K, Mishiro T. Cohesin mediates transcriptional insulation by CCCTC-binding factor. Nature, 2008, 451(7180): 796-1801.[DOI]

\section{•综合信息・}

\section{国际生物化学与分子生物学大会将在上海召开}

“ $21^{\text {st }}$ IUBMB and $12^{\text {th }}$ FAOBMB International Congress of Biochemistry and Molecular Biology” (第 21 届国际生物化 学与分子生物学联盟学术大会暨第 12 届亚洲大洋洲生物化学家与分子生物学家学术大会, 简称国际生化大会)将于 2009 年 8 月 2 7 日在上海国际会议中心召开。国际生化大会每 3 年举办 1 次, 是国际生命科学研究领域最重要的序列 会议之一, 是首次在我国举办。中国生物化学与分子生物学会和中国细胞生物学学会将共同承办这次盛会。

国际生化大会将提供生命科学最前沿的研究成果。它涵盖了生命科学中的 4 大主题(基因组动态和基因调控; 蛋白 质结构、动态与蛋白质组; 细胞信号网络; 疾病的分子机制), 共有 36 个专题分会场; 此外, 大会还包括一些其他专题 会议，如糖生物学、脂生物学、生物技术与技术转移等(详细内容见 www.iubmb-faobmb2009.cn)。

大会已经邀请并落实了众多来自世界各地的优秀科学家在会议上做报告。12 位顶尖科学家已经确认到会做大会主 题报告, 包括 4 位诺贝尔奖获得者以及发现 iPS 细胞的 Dr. Shinya Yamanaka 和因发现 microRNA 获得 2008 年 Lasker 奖 的 Dr. Victor Ambros。与此同时, 160 余位在各自研究领域做出杰出贡献的科学家已经同意到会在相关的学术专题会上 做学术报告。据对这些同意参加会议的报告人的不完全统计，拥有正教授职称的会议报告人已经超过 $80 \%$ ，其中来自美 国的正教授有 60 多人(包括 8 个 HHMI 的研究员)，来自欧洲的正教授有 30 多人，来自世界其他地区的正教授有 20 多人。

大会组委会邀请国内广大研究人员、老师、研究生和博士后参加这次生命科学的盛会。在线注册从 2009 年 1 月 1 日已经正式开通。您可以登陆大会网站(www.iubmb-faobmb2009.cn)进行在线注册，并获取更多关于此次会议的信息。 优惠在线注册的时间是 1 月 1 日到 4 月 30 日。正常在线注册的时间是 5 月 1 日到 6 月 30 日。在线注册和论文提交的 截止日期为 2009 年 7 月 1 日。 